Z.-Q. Chen, Z.-M. Ma and M. Röckner

Nagoya Math. J.

Vol. 136 (1994), 1-15

\title{
QUASI-HOMEOMORPHISMS OF DIRICHLET FORMS
}

\author{
ZHEN-QING CHEN, ZHI-MING MA AND MICHAEL RÖCKNER
}

\section{\$1. Introduction}

Extending fundamental work of M. Fukushima, M. L. Silverstein, S. Carrillo Menendez, and Y. Le Jan (cf. [F71a, 80], [Si74], [Ca-Me75], [Le77]) it was recently discovered that there is a one-to-one correspondence between (equivalent classes of) all pairs of sectorial right processes and quasi-regular Dirichlet forms (see [AM91], [AM92], [AMR90], [AMR92a], [AMR92b], [MR92]). Based on the potential theory for quasi-regular Dirichlet forms, it was shown that any quasi-regular Dirichlet form on a general state space can be considered as a regular Dirichlet form on a locally compact separable metric space by "local compactification". There are several ways to implement this local compactification. One relies on $h$-transformation which was mentioned in [AMR90, Remark 1.4]. A direct way using a modified Ray-Knight compactification was announced on the " 5 th French-German meeting: Bielefeld Encounters in Mathematics and Physics IX. Dynamics in Complex and Irregular Systems", Bielefeld, December 16 to 21, 1991, and the "Third European Symposium on Analysis and Probability", Paris, January 3-10, 1992, and appeared in [MR92, Chap. VI] and [AMR92b] (see also the proof of Theorem 3.7 below). One can also do this by Gelfand-transform. This way was found by the first named author independetly and announced in the "12th Seminar on Stochastic Processes", Seattle, March 26-28, 1992. It will be discussed in Section 4.

The main purpose of this paper is to take another point of view to look upon quasi-regular Dirichlet forms. We shall define a concept of quasi-homeomorphism for Dirichlet forms in terms of $\mathscr{E}$-nests (see Definition 3.1 below). Using this concept we may rephrase the local compactification result as that every quasi-regular Dirichlet form is quasi-homeomorphic to a regular Dirichlet form. But one can see that the converse is also true. Therefore, from this point of view a Dirichlet form is quasi-regular if and only if it is quasi-homeomorphic to a regular Dirichlet

Received July 9, 1993. 
form on a locally compact separable metric space. See Theorem 3.7 for details. We mention that our concept of quasi-homeomorphism is a modification of the one used in [F71a] and [Si74] where the same concept was defined in terms of the classical capacity and hence cannot be used directly for general Dirichlet forms (see Remark 3.2).

The remainder of this paper is organized as follows. In Section 2 we recall some basic facts on quasi-regular Dirichlet forms which are necessary for the subsequent discussion. Section 3 is devoted to the main results concerning quasihomeomorphism of Dirichlet forms. In Section 4 we describe the above mentioned "Gelfand-transform approach" to local compactification, whose spirit can be traced back to Fukushima's regular representation theory [F71b].

\section{§2. Basics on quasi-regular Dirichlet forms}

We first recall some basic potential theory for general Dirichlet forms. They appeared in [AMR90], [AM91] for the symmetric case and were developed in detail in [MR92] (see also [AMR92a, b]) for the general (non-symmetric) case. For convenience from now on we shall refer only to [MR92], and only use the terminology therein.

In what follows let $E$ be a Hausdorff topological space with $\sigma$-algebra $\mathscr{B}(E)$. Let $m$ be a $\sigma$-finite positive measure on $(E, \mathscr{B}(E))$. Let $(\mathscr{E}, D(\mathscr{E}))$ be a fixed (not necessarily symmetric) Dirichlet form on $L^{2}(E ; m)$ with associated generator $L$, semigroups $\left(T_{t}\right)_{t>0},\left(\hat{T}_{t}\right)_{t>0}$ and resolvents $\left(G_{\alpha}\right)_{\alpha>0},\left(\hat{G}_{\alpha}\right)_{\alpha>0}$ on $L^{2}(E ; m)$. For $\alpha>0$ we shall write $\mathscr{E}_{\alpha}(u, v):=\mathscr{E}(u, v)+\alpha(u, v)$ for $u, v \in$ $D(\mathscr{E})$, where $($,$) is the usual inner product in L^{2}(E ; m)$. Let $\widetilde{\mathscr{E}}$ be the symmetric part of $\mathscr{E}$, i.e., $\tilde{\mathscr{E}}(u, v):=\frac{1}{2}[\mathscr{E}(u, v)+\mathscr{E}(v, u)]$. Then $(\widetilde{\mathscr{E}}, D(\mathscr{E}))$ is a symmetric

Dirichlet form on $L^{2}(E ; m)$. The associated objects relative to $(\tilde{\mathscr{E}}, D(\mathscr{E}))$ will be marked by " ", e.g. $\left(\tilde{G}_{\alpha}\right)_{\alpha>0}$ will denote the resolvent associated with $(\tilde{\mathscr{E}}, D(\mathscr{E}))$. Let $K \geq 1$ be a continuity constant of $(\mathscr{E}, D(\mathscr{E}))$, i.e., $K$ satisfies $\left|\mathscr{E}_{1}(u, v)\right|$ $\leq K \mathscr{E}_{1}(u, u)^{1 / 2} \mathscr{E}_{1}(v, v)^{1 / 2}$ for all $u, v \in D(\mathscr{E})$.

For a closed set $F \subset E$, we define

$$
D(\mathscr{E})_{F}:=\{u \in D(\mathscr{E}) \mid u=0 \text { m-a.e. on } E \backslash F\} .
$$

Definition 2.1. (i) An increasing sequence $\left(F_{k}\right)_{k \in \mathbf{N}}$ of closed subsets of $E$ is called an $\mathscr{E}$-nest if $\cup_{k \geq 1} D(\mathscr{E})_{F_{k}}$ is dense in $D(\mathscr{E})$ with respect to $\tilde{\mathscr{E}}_{1}^{1 / 2}$-norm.

(ii) A subset $N \subset E$ is called $\mathscr{E}$-exceptional if $N \subset \cap_{k \geq 1}\left(E \backslash F_{k}\right)$ for some 
$\mathscr{E}$-nest $\left(F_{k}\right)_{k \in \mathbf{N}}$. We say that a property of points in $E$ holds $\mathscr{E}$-quasieverywhere (abbreviated $\mathscr{E}$-q.e.), if the property holds outside some $\mathscr{E}$-exceptional set.

For a given $\mathscr{E}$-nest $\left(F_{k}\right)_{k \in \mathbf{N}}$ we define

$$
C\left(\left\{F_{k}\right\}\right):=\left\{f: A \rightarrow R \mid \bigcup_{k \geq 1} F_{k} \subset A \subset E, f_{\mid F_{k}} \text { is continuous for all } k\right\} .
$$

Definition 2.2. An $\mathscr{E}$-q.e. defined function $f$ on $E$ is called $\mathscr{E}$-quasicontinuous if there exists an $\mathscr{E}$-nest $\left(F_{k}\right)_{k \in \mathbf{N}}$ such that $f \in C\left(\left\{F_{k}\right\}\right)$.

The above notions can also be characterized by certain (equivalent) capacities relative to $(\mathscr{E}, D(\mathscr{E}))$. To this end we set

$$
\mathscr{H}:=\left\{h \in D(\mathscr{g}) \mid h=\tilde{G}_{1} \varphi \text { for some } \varphi \in L^{2}(E ; m), 0<\varphi \leq 1 \text { m-a.e. }\right\} .
$$

For $h \in \mathscr{H}$, we define for open $U \subset E$,

$$
\operatorname{Cap}_{h}(U):=\inf \left\{\mathscr{E}_{1}(u, u) \mid u \in D(\mathscr{E}), u \geq h \text { m-a.e. on } U\right\} .
$$

and for arbitrary $A \subset E$,

$$
\operatorname{Cap}_{h}(A):=\inf \left\{\operatorname{Cap}_{h}(U) \mid A \subset U \subset E, U \text { open }\right\} .
$$

Remark 2.3. For $h \in \mathscr{H}$ and $U \subset E, U$ open, there exists a unique $\tilde{h}_{U} \in$ $D(\mathscr{E})$, called the 1 -reduced function of $h$ (relative to $\widetilde{\mathscr{E}}$ ), such that $0 \leq \tilde{h}_{U} \leq h$ m-a.e., $\tilde{h}_{U}=h$ m-a.e. on $U$ and $\operatorname{Cap}_{h}(U)=\mathscr{E}_{1}\left(\tilde{h}_{U}, \tilde{h}_{U}\right.$ ) (cf. [MR92, III1.5 and 2.10])

Theorem 2.4. Let $h \in \mathscr{H}$. Then $\mathrm{Cap}_{h}$ is a Choquet capacity which is countably subadditive, $i$. e., it has the following properties:

( i ) $A_{n} \uparrow A \Rightarrow \operatorname{Cap}_{h}\left(A_{n}\right) \uparrow \operatorname{Cap}_{h}(A)$.

(ii) $K_{n} \downarrow K, K_{n}$ compact $\Rightarrow \operatorname{Cap}_{h}\left(K_{n}\right) \downarrow \operatorname{Cap}_{h}(K)$.

(iii) $\operatorname{Cap}_{h}\left(\cup_{n \geq 1}^{\cup} A_{n}\right) \leq \sum_{n \geq 1} \operatorname{Cap}_{h}\left(A_{n}\right)$.

Proof. See [MR92, III. 2.8].

THEOREM 2.5. (i) An increasing sequence $\left(F_{k}\right)_{k \in \mathbf{N}}$ of closed subsets of $E$ is an $\mathscr{E}$-nest if and only if $\lim _{k \rightarrow \infty} \operatorname{Cap}_{h}\left(E \backslash F_{k}\right)=0$.

(ii) A subset $N \subset E$ is $\mathscr{E}$-exceptional if and only if $\operatorname{Cap}_{h}(N)=0$. 
Proof. See [MR92, III. 2.11].

By Theorem 2.5 the capacities $\mathrm{Cap}_{h}, h \in \mathscr{H}$, are equivalent to each other.

By virtue of Theorem 2.5, one can prove the following lemma analogously to [F80, Theorem 3.1.2(i)].

Lemma 2.6 [MR92, III. 3.3]. Let $S$ be a countable family of $\mathscr{E}$-quasi-continuous functions on $E$. Then there exists an $\mathscr{E}$-nest $\left(F_{k}\right)_{k \in \mathbf{N}}$ such that $S \subset C\left(\left\{F_{k}\right\}\right)$.

Let $f_{n}, n \in \mathbf{N}$, be (E्E-q.e. defined) functions on $E$. We say that $\left(f_{n}\right)_{n \in \mathbf{N}}$ converges to $f \mathscr{E}$-quasi-uniformly if there exists an $\mathscr{E}$-nest $\left(F_{k}\right)_{k \in \mathbf{N}}$ such that $f_{n} \rightarrow f$ uniformly on each $F_{k}$. The followung result relies also heavily on Theorem 2.5 (cf. [F80, Theorem 3.1.4]).

PROPOSITION 2.7 [MR92, III. 3.5]. Let $u_{n} \in D(\mathscr{E})$, which have $\mathscr{E}$-quasicontinuous $m$-versions $\tilde{u}_{n}, n \in \mathbf{N}$, such that $u_{n} \rightarrow u \in D(\mathscr{E})$ with respect to $\tilde{\mathscr{E}}_{1}^{1 / 2}$-norm. Then there exists a subsequence $\left(u_{n_{k}}\right)_{k \in \mathbf{N}}$ and an $\mathscr{E}$-quasi-continuous $m$-version $\tilde{u}$ of $u$ such that $\left(\tilde{u}_{n_{k}}\right)_{k \in \mathbf{N}}$ converges $\mathscr{E}$-quasi-uniformly to $\tilde{u}$.

We are now prepared to recall the following definition.

Definition 2.8. $(\mathscr{E}, D(\mathscr{E}))$ is called a quasi-regular Dirichlet form if:

(i) There exists an $\mathscr{E}$-nest $\left(E_{k}\right)_{k \in \mathbf{N}}$ consisting of compact sets.

(ii) There exists an $\widetilde{\mathscr{E}}_{1}^{1 / 2}$-dense subset of $D(\mathscr{E})$ whose elements have $\mathscr{E}$-quasicontinuous $m$-versions.

(iii) There exist $u_{n} \in D(\mathscr{E}), n \in \mathbf{N}$, having $\mathscr{E}$-quasi-continuous m-versions $\tilde{u}_{n}$, $n \in \mathbf{N}$, and an $\mathscr{E}$-exceptional set $N \subset E$ such that $\left\{\tilde{u}_{n} \mid n \in \mathbf{N}\right\}$ separates the points of $E \backslash N$.

In what follows we collect some basic properties of quasi-regular Dirichlet forms whose detailed proofs can be found in [MR92, IV. §3].

Proposition 2.9. Let $(\mathscr{E}, D(\mathscr{E}))$ be a quasi-regular Dirichlet form on $L^{2}(E ; m)$. Then:

(i) $D(\mathscr{E})$ is separable with respect to $\widetilde{\mathscr{E}}_{1}^{1 / 2}$.

(ii) Each element $u \in D(\mathscr{E})$ has an $\mathscr{E}$-quasi-continuous $m$-version $\tilde{u}$.

(iii) If $\left(F_{k}\right)_{k \in \mathbf{N}}$ is an $\mathscr{E}-$ nest and $F_{k}^{\prime}=\operatorname{supp}\left[I_{F_{k}} \cdot m\right]$, then $\left(F_{k}^{\prime}\right)_{k \in \mathbf{N}}$ is an $\mathscr{E}$-nest. 
(iv) If $f$ is $\mathscr{E}$-quasi-continuous and $f \geq 0$ m-a.e. on an open set $U$ of $E$, then $f \geq 0$ $\mathscr{E}$-q.e. on $U$. In particular, $\tilde{u}$ is $\mathscr{E}$-q.e. unique for all $u \in D(\mathscr{E})$.

(v) If $D$ is a set of $\mathscr{E}$-quasi-continuous functions such that the corresponding $m$-classes form a dense subset of $D(\mathscr{E})$, then there exists an $\mathscr{E}$-exceptional set $N$ such that $D$ separates the points of $E \backslash N$.

(vi) Let $h$ be an $\mathscr{E}$-quasi-continuous $m$-version of an element in $\mathscr{H}$ (H being specified by (2.3)), then there exists an $\mathscr{E}-$ nest $\left(F_{k}^{h}\right)_{k \in \mathbf{N}}$ consisting of compact metrizable sets such that

$$
\inf \left\{h(x) \mid x \in F_{k}^{h}\right\}>0 \text { for all } k \in \mathbf{N}
$$

The following is crucial for the discussion below.

Proposition 2.10. Let $(\mathscr{E}, D(\mathscr{E}))$ be a quasi-regular Dirichlet form on $L^{2}(E ; m)$. Then there exists a countable set $D$ of $\mathscr{E}$-quasi-continuous functions such that the corresponding $m$-classes form a dense subset of $D(\mathscr{E})$ satisfying the following properties:

(i) $D$ is an algebra over the rationals and $h^{2} \in D$, where $h$ is specified by 2.9 (vi).

(ii) Each $u$ in $D$ is bounded and $|u| \leq c h^{2}$ for some $c>0$. In particular, each $u \in D$ is m-integrable.

(iii) There exists an $\mathscr{E}$-nest $\left(F_{k}\right)_{k \in \mathbf{N}}$ such that $D \subset C\left(\left\{F_{k}\right\}\right), D$ separates the points of $Y:=\cup F_{k}$, and $F_{k} \subset F_{k}^{h}$ with $F_{k}^{h}$ being specified by 2.9(vi). Moreover, $F_{k}=$ $\operatorname{supp}\left[I_{F_{k}} \cdot m\right]$ for each $k$.

Proof. Since $\cup_{k \geq 1} D(\mathscr{E})_{F_{k}^{h}}$ is dense and $D(\mathscr{E})$ is separable with respect to $\tilde{\mathscr{E}}_{1}^{1 / 2}$-norm, we may take a countable subset $D_{1} \subset \cup_{k \geq 1} D(\mathscr{E})_{F_{k}^{h}}$ such that $D_{1}$ is $\tilde{\mathscr{E}}^{1 / 2}$-dense in $D(\mathscr{E})$. Moreover, since $(u \wedge n) \vee(-n) \rightarrow u$ in $\mathscr{\mathscr { E }}^{1 / 2}$-norm for each $u \in D(\mathscr{E})$, we may assume that each element of $D_{1}$ is bounded. For each $u \in D_{1}$, we fix an $\mathscr{E}$-quasi-continuous m-version $\tilde{u}$ such that

$$
\tilde{u}=0 \text { on } E \backslash F_{k}^{h} \text { for some } k \in \mathbf{N} \text {. }
$$

Let $D$ be the smallest algebra over the rationals which is generated by $h^{2}$ and $\left\{\tilde{u} \mid u \in D_{1}\right\}$. Then $D$ is a countable dense subset of $D(\mathscr{E})$ satisfying 2.10 (i) and (ii). By 2.8 (v) there is an $\mathscr{E}$-exceptional set $N$ such that $D$ separates the points of $E \backslash N$. Let $\left(F_{1 k}\right)_{k \in \mathbf{N}}$ be an $\mathscr{E}$-nest such that $N \subset \cap_{k \geq 1}\left(E \backslash F_{1 k}\right)$. Let $\left(F_{2 k}\right)_{k \in \mathbf{N}}$ be an $\mathscr{E}$-nest such that $D \subset C\left(\left\{F_{2 k}\right\}\right)$. Let $F_{k}^{\prime}:=F_{1 k} \cap F_{2 k} \cap F_{k}^{h}$ and $F_{k}:=$ $\operatorname{supp}\left[I_{F_{k}^{\prime}} \cdot m\right]$. Then $\left(F_{k}\right)_{k \in \mathbf{N}}$ is an $\mathscr{E}$-nest satisfying 2.10 (iii). 


\section{§3. Quasi-homeomorphisms of Dirichlet forms}

Let $(\mathscr{E}, D(\mathscr{E}))$ be a Dirichlet form on $L^{2}(E ; m)$ as in the previous section. Let $E^{\#}$ be another Hausdorff topological space with Borel $\sigma$-algebra $\mathscr{B}\left(E^{\#}\right)$. Let $Y:=E \backslash N$ where $N \in \mathscr{B}(E)$ is an $\mathscr{E}$-exceptional set. Suppose that $j$ is a $\mathscr{B}(Y) /$ $\mathscr{B}\left(E^{\#}\right)$-measurable map from $Y$ into $E^{\#}$. Let $m^{\circ} j^{-1}$ be the image measure of $m$ on

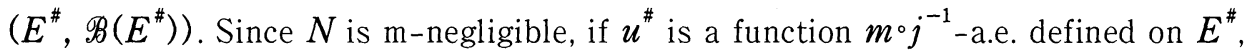
then $u^{\#} \circ j$ is m-a.e. defined on $E$. Therefore, if we set

$$
j^{*} u^{\#}:=u^{\#} \circ j \mathrm{~m} \text {-a.e. for } u^{\#} \in L^{2}\left(E^{\#} ; m^{\circ} j^{-1}\right),
$$

then $j^{*}$ is an isometric map from $L^{2}\left(E^{\#} ; m^{\circ} j^{-1}\right)$ into $L^{2}(E ; m)$. In this paper we use the same notation for a function $f$ (m-a.e. defined) on $E$ and for the m-equivalence class of functions represented by $f$, if there is no risk of confusion. We define

$$
\left\{\begin{array}{l}
D\left(\mathscr{E}^{j}\right):=\left\{u^{\#} \in L^{2}\left(E^{\#} ; m^{\circ} j^{-1}\right) \mid j^{*} u^{\#} \in D(\mathscr{\mathscr { E }})\right\} \\
\mathscr{E}^{j}\left(u^{\#}, v^{\#}\right):=\mathscr{E}\left(j^{*} u^{\#}, j^{*} v^{\#}\right) ; u^{\#}, v^{\#} \in D\left(\mathscr{E}^{j}\right) .
\end{array}\right.
$$

$\left(\mathscr{E}^{j}, D\left(\mathscr{E}^{j}\right)\right)$ is called the image of $(\mathscr{E}, D(\mathscr{E}))$ under $j$. Clearly, $\left(\mathscr{E}^{j}, D\left(\mathscr{E}^{j}\right)\right)$ is a Dirichlet form if $j^{*}$ is onto.

In what follows let $m^{\#}$ be a $\sigma$-finite Borel measure on $E^{\#}$. Let $(\mathscr{E}, D(\mathscr{E}))$ and $\left(\mathscr{E}^{\#}, D\left(\mathscr{E}^{\#}\right)\right)$ be two Dirichlet forms on $L^{2}(E ; m)$ and $L^{2}\left(E^{\#} ; m^{\#}\right)$ respectively. All the notions relative to $\left(\mathscr{E}^{\#}, D\left(\mathscr{E}^{\#}\right)\right)$ will be marked by “\#”, e.g., $\left(\widetilde{\mathscr{E}}^{\#}, D\left(\mathscr{E}^{\#}\right)\right)$ denotes the symmetric part of $(\mathscr{E}, D(\mathscr{E})),\left(G_{\alpha}^{\#}\right)_{\alpha>0}\left(\right.$ resp. $\left.\left(\hat{G}_{\alpha}^{\#}\right)_{\alpha>0}\right)$ denotes the resolvent associated with $\left(\mathscr{E}^{\#}, D\left(\mathscr{E}^{\#}\right)\right)\left(\right.$ resp. $\left(\mathscr{\mathscr { E }}^{\#}, D\left(\mathscr{E}^{\#}\right)\right)$ etc.

Definition 3.1. $(\mathscr{E}, D(\mathscr{E}))$ is said to be quasi-homeomorphic to $\left(\mathscr{E}^{\#}, D\left(\mathscr{E}^{\#}\right)\right)$, if there is a map $j: \cup_{k \geq 1} F_{k} \rightarrow \cup_{k \geq 1} F_{k}^{\#}$, where $\left(F_{k}\right)_{k \in \mathbf{N}}$ is an $\mathscr{E}$-nest in $E$ and $\left(F_{k}^{*}\right)_{k \in \mathbf{N}}$ an $\mathscr{E}^{\#}$-nest in $E^{\#}$, such that:

(i) $j$ is a topological homeomorphism from $F_{k}$ onto $F_{k}^{\#}$ for each $k \in \mathbf{N}$.

(ii) $m^{\#}=m^{\circ} j^{-1}$, where $m^{\circ} j^{-1}$ is the image measure of $m$ under $j$.

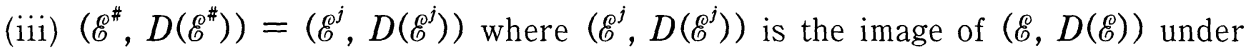
$j$ defined by (3.2).

Such a map $j$ is called a quasi-homeomorphism from $(\mathscr{E}, D(\mathscr{E}))$ to $\left(\mathscr{E}^{\#}, D\left(\mathscr{E}^{\#}\right)\right)$.

Remark 3.2. The above definition of quasi-homeomorphism is a modification of the one used in [Fu71a] and [Si74]. There the same concept was defined in terms of classical capacities for regular Dirichlet forms, and hence it is not directly applicable to general Dirichlet forms, since for a general Dirichlet form 
the classical capacity may be identically infinite for any non-empty subset (cf.[MR92,II.2.e)] for an example of such a Dirichlet form).

Lemma 3.3. Suppose that $(\mathscr{E}, D(\mathscr{E}))$ is quasi-homeomorphic to $\left(\mathscr{E}^{\#}, D\left(\mathscr{E}^{\#}\right)\right)$, and let $j$ be the corresponding quasi-homeomorphism. Then:

(i ) $\left(\mathscr{E}^{\#}, D\left(\mathscr{E}^{\#}\right)\right)$ is quasi-homeomorphic to $(\mathscr{E}, D(\mathscr{E}))$ and $j^{-1}$ is a quasihomeomorphism from $\left(\mathscr{E}^{\#}, D\left(\mathscr{E}^{\#}\right)\right)$ to $(\mathscr{E}, D(\mathscr{E}))$.

(ii) $j^{*} \operatorname{maps} L^{2}\left(E^{\#} ; m^{\#}\right)$ onto $L^{2}(E ; m)$ and hence $L^{2}\left(E^{\#} ; m^{\#}\right)$ is isomorphic to $L^{2}(E ; m)$.

(iii) $j^{*}$ maps $D\left(\mathscr{E}^{\#}\right)$ onto $D(\mathscr{E})$ and hence the two Hilbert spaces $\left(D\left(\mathscr{E}^{*}\right), \widetilde{\mathscr{E}}_{1}^{\#}\right)$ and $\left(D(\mathscr{E}), \widetilde{\mathscr{E}}_{1}\right)$ are isomorphic.

(iv) For $\alpha>0$ we have

$$
\begin{aligned}
& G_{\alpha}\left(j^{*} f^{\#}\right)=j^{*} G_{\alpha}^{\#} f^{\#} \text { for all } f^{\#} \in L^{2}\left(E^{\#} ; m^{\#}\right) \\
& \tilde{G}_{\alpha}\left(j^{*} f^{\#}\right)=j^{*} \tilde{G}_{\alpha}^{\#} f^{\#} \text { for all } f^{\#} \in L^{2}\left(E^{\#} ; m^{\#}\right) .
\end{aligned}
$$

Proof. (i) can be checked in a straightforward way by definition. (ii) and (iii) are consequences of (i). For proving (iv) we only have to show (3.3). Denote by $(\cdot, \cdot)$ and $(\cdot, \cdot)_{\#}$ the inner products of $L^{2}(E ; m)$ and $L^{2}\left(E^{\#} ; m^{\#}\right)$ respectively. For $f^{\#} \in L^{2}\left(E^{\#} ; m^{\#}\right)$ and $g^{\#} \in D\left(\mathscr{E}^{\#}\right)$, we have

$$
\begin{aligned}
& \mathscr{E}_{\alpha}\left(G_{\alpha}\left(j^{*} f^{\#}\right), j^{*} g^{\#}\right)=\left(j^{*} f^{\#}, j^{*} g^{\#}\right)=\left(f^{\#}, g^{\#}\right)_{\#,}, \\
& \mathscr{E}_{\alpha}\left(j^{*} G_{\alpha}^{\#} f^{\#}, j^{*} g^{\#}\right)=\mathscr{E}_{\alpha}^{\#}\left(G_{\alpha}^{\#} f^{\#}, g^{\#}\right)=\left(f^{\#}, g^{\#}\right)_{\#} .
\end{aligned}
$$

Therefore, since $j^{*}$ maps $D\left(\mathscr{E}^{\#}\right)$ onto $D(\mathscr{E})$, we obtain for $f^{\#} \in L^{2}\left(E^{\#} ; m^{\#}\right)$ that $\mathscr{E}_{\alpha}\left(G_{\alpha}\left(j^{*} f^{\#}\right), g\right)=\mathscr{E}_{\alpha}\left(j^{*} G_{\alpha}^{\#} f^{\#}, g\right)$ for all $g \in D(\mathscr{E})$.

Thus by the strict positivity of $\mathscr{E}_{\alpha}$ we get $G_{\alpha}\left(j^{*} f^{\#}\right)=j^{*} G_{\alpha}^{\#} f^{\#}$.

Remark 3.4. (i) Lemma 3.3 (i) shows that the quasi-homeomorphism relation is symmetric. Clearly it is also transitive, hence the quasihomeomorphism relation is an equivalence relation among Dirichlet forms on Hausdorff state spaces.

(ii) Lemma 3.3 (ii)-(iv) show that a quasi-homeomorphism keeps the Hilbert structures of $L^{2}(E ; m)$ and $\left(D(\mathscr{E}), \widetilde{\mathscr{E}}_{1}\right)$ invariant. In Theorem 3.5 and Corollary 3.6 below we shall show that a quasi-homeomorphism keeps also the potential theoretic properties of a Dirichlet form invariant.

Let $\mathscr{H}$ and $\mathscr{H}^{\#}$ be specified by $(2.3)$ relative to $(\mathscr{E}, D(\mathscr{E}))$ and $\left(\mathscr{E}^{\#}, D\left(\mathscr{E}^{\#}\right)\right)$ respectively. 
THEOREM 3.5. Suppose that $(\mathscr{E}, D(\mathscr{E}))$ is quasi-homeomorphic to $\left(\mathscr{E}^{\#}, D\left(\mathscr{E}^{\#}\right)\right)$ and let $j: \cup_{k \geq 1} F_{k} \rightarrow \cup_{k \geq 1} F_{k}^{\#}$ be the quasi-homeomorphism. Let $h^{\#} \in \mathscr{H}^{\#}$ and $h:=$ $j^{*} h^{\#}$, then $h \in \mathscr{H}$. Moreover, if $Y:=\cup_{k \geq 1} F_{k}$ and $Y^{\#}:=\cup_{k \geq 1} F_{k}^{\#}$, then

$$
\operatorname{Cap}_{h}(B)=\operatorname{Cap}_{h^{*}}^{\#}(j(Y \cap B)) \text { for all } B \subset E \text {, }
$$

or equivalently,

$$
\operatorname{Cap}_{h^{\#}}^{\#}\left(B^{\#}\right)=\operatorname{Cap}_{h}\left(j^{-1}\left(Y^{\#} \cap B^{\#}\right)\right) \text { for all } B^{\#} \subset E^{\#} .
$$

Proof. Assume that $h^{\#}=\tilde{G}_{1}^{\#} \varphi^{\#}$ for some $\varphi^{\#} \in L^{2}\left(E^{\#} ; m^{\#}\right), 0<\varphi^{\#} \leq 1 \mathrm{~m}^{\#}$-a.e. Let $\varphi:=j^{*} \varphi^{*}$, then $0<\varphi \leq 1 \mathrm{~m}$-a.e. Therefore, by (3.4) $h=j^{*} h^{*}=\widetilde{G}_{1} \varphi \in \mathscr{H}$. For proving (3.5) we take an arbitrary $\delta>0$. Let $B \subset E$ and set $B^{\#}:=j(Y \cap B)$. Take an open set $U^{\#} \subset E^{\#}$ such that $B^{\#} \subset U^{\#}$ and $\operatorname{Cap}_{h^{*}}^{\#}\left(U^{\#}\right) \leq \operatorname{Cap}_{h^{\#}}^{\#}\left(B^{\#}\right)+$ $\delta$. Set $f^{\#}:=\tilde{h}_{U^{\#}}^{\#}$, where $\tilde{h}_{U^{\#}}^{\#}$ is the 1 -reduced function of $h^{\#}$ relative to $\left(\tilde{\mathscr{E}}^{\#}, D\left(\mathscr{E}^{\#}\right)\right.$ ) (cf. Remark 2.3). Let $f:=j^{*} f^{\#}$, then $0 \leq f \leq h$ m-a.e. and $f \geq h$ m-a.e. on $j^{-1}\left(Y^{\#} \cap U^{\#}\right) \supset Y \cap B$. By Theorem 2.5 we may take $n \in \mathbf{N}$ such that $\operatorname{Cap}_{h}\left(E \backslash F_{n}\right) \leq \delta$. Let $f_{n}:=f+\tilde{h}_{E \backslash F_{n}}$, where $\tilde{h}_{E \backslash F_{n}}$ is the 1 -reduced function of $h$ relative to $(\widetilde{\mathscr{E}}, D(\mathscr{E}))$. Then $f_{n} \geq h \mathrm{~m}$-a.e. on $U_{n}:=j^{-1}\left(Y^{\#} \cap U^{\#}\right) \cup\left(E \backslash F_{n}\right)$. Since $U_{n}$ is an open set containing $B$, we obtain

$$
\begin{aligned}
\operatorname{Cap}_{h}(B) & \leq \operatorname{Cap}_{h}\left(U_{n}\right) \leq \mathscr{E}_{1}\left(f_{n}, f_{n}\right) \leq\left[\mathscr{E}_{1}(f, f)^{1 / 2}+\operatorname{Cap}_{h}\left(E \backslash F_{n}\right)^{1 / 2}\right]^{2} \\
& \leq\left[\mathscr{E}_{1}^{\#}\left(f^{\#}, f^{\#}\right)^{1 / 2}+\delta^{1 / 2}\right]^{2} \leq\left[\left(\operatorname{Cap}_{h}^{\#}\left(B^{\#}\right)+\delta\right)^{\frac{1}{2}}+\delta^{1 / 2}\right]^{2} .
\end{aligned}
$$

Letting $\delta \downarrow 0$ we obtain

$$
\operatorname{Cap}_{h}(B) \leq \operatorname{Cap}_{h^{*}}^{\#}\left(B^{\#}\right)=\operatorname{Cap}_{h^{*}}^{\#}(j(Y \cap B)) .
$$

By Lemma 3.3 (i) we may interchange the roles of $(\mathscr{E}, D(\mathscr{E}))$ and $\left(\mathscr{E}^{\#}, D\left(\mathscr{E}^{\#}\right)\right)$ to obtain

$$
\operatorname{Cap}_{h^{\#}}^{\#}(j(Y \cap B)) \leq \operatorname{Cap}_{h}\left(j^{-1} j(Y \cap B)\right)=\operatorname{Cap}_{h}(Y \cap B)=\operatorname{Cap}_{h}(B),
$$

where we used the fact that $\operatorname{Cap}_{h}(E \backslash Y)=0$ in the last step. Thus (3.5) is proved.

COROLLARY 3.6. In the situation of Theorem 3.5, the following statements hold.

(i ) Let $\left(E_{n}\right)_{n \in \mathbf{N}}$ be an increasing sequence of closed subsets of $E$. Then $\left(E_{n}\right)_{n \in \mathbf{N}}$ is an $\mathscr{E}-$ nest if and only if $\left(j\left(F_{n} \cap E_{n}\right)\right)_{n \in \mathbf{N}}$ is an $\mathscr{E}^{\#}-$ nest.

(ii) $N \subset E$ is $\mathscr{E}$-exceptional if and only if $j(Y \cap N)$ is $\mathscr{E}^{\#}$-exceptional.

(iii) A function $f, \mathscr{E}$-q.e. defined on $E$, is $\mathscr{E}$-quasi-continuous if and only if $f_{\circ} j^{-1}$ is $\mathscr{E}^{\#}$-quasi-continuous. 
Proof. (i) follows from Theorem 3.3 and Theorem 2.5. (ii) and (iii) are consequences of (i).

Recall that a Dirichlet form $(\mathscr{E}, D(\mathscr{E}))$ on $L^{2}(E ; m)$ is regular (cf. [Si74], [F80]) if it satisfies the following three conditions:

(3.7) $E$ is locally compact separable metric space and $m$ is a positive Radon measure on $E$ with $\operatorname{supp}[m]=E$.

(3.8) $\quad C_{0}(E) \cap D(\mathscr{E})$ is dense in $D(\mathscr{E})$ with respect to $\widetilde{\mathscr{E}}^{1 / 2}$. (Here and henceforth $C_{0}(E)$ is the family of all continuous functions with compact support on $E$.) $C_{0}(E) \cap D(\mathscr{E})$ is dense in $C_{0}(E)$ with respect to the uniform norm.

Now we can prove our main result.

Theorem 3.7. A Dirichlet form $(\mathscr{E}, D(\mathscr{E}))$ on $L^{2}(E ; m)$ is quasi-regular if and only if it is quasi-homeomorphic to a regular Dirichlet form $\left(\mathscr{E}^{\#}, D\left(\mathscr{E}^{\sharp}\right)\right)$ on $L^{2}\left(E^{\#} ; m^{\#}\right)$.

Proof. (i) "if"-part: Suppose that $(\mathscr{E}, D(\mathscr{E}))$ is quasi-homeomorphic to a regular Dirichlet form $\left(\mathscr{E}^{\#}, D\left(\mathscr{E}^{\#}\right)\right)$ on $L^{2}\left(E^{\#} ; m^{\#}\right)$, and $j: \cup_{k \geq 1} F_{k} \rightarrow \cup_{k \geq 1} F_{k}^{\#}$ is a quasi-homeomorphism, where $\left(F_{k}\right)_{k \in \mathbf{N}}$ is an $\mathscr{E}$-nest and $\left(F_{k}^{\#}\right)_{k \in \mathbf{N}}$ an $\mathscr{E}^{\#}$-nest. We need to show that $(\mathscr{E}, D(\mathscr{E}))$ satisfies 2.8 (i)-(iii). Let $\left(U_{n}^{\#}\right)_{n \in \mathbf{N}}$ be an increasing sequence of relatively compact open sets with $\cup_{k \geq 1} U_{k}^{\#}=E^{\#}$. Then $E_{k}^{\#}:=\bar{U}_{k}^{\#}$ is compact and $\left(E_{k}^{\#}\right)_{k \in \mathbf{N}}$ is an $\mathscr{E}^{\#}$-nest since $C_{0}\left(E^{\#}\right) \cap D\left(\mathscr{E}^{\#}\right) \subset \cup_{k \geq 1} D\left(\mathscr{E}^{\#}\right)_{E_{k}^{*}}$. Let $E_{k}:=j^{-1}\left(E_{k}^{\#} \cap F_{k}^{\#}\right)$. Then each $E_{k}$ is compact and by 3.6 (i) $\left(E_{k}\right)_{k \in \mathbf{N}}$ is an $\mathscr{E}$-nest, and 2.8 (i) is verified. Let $D:=\left\{u=u^{\#} \circ j \mid u^{\#} \in C_{0}\left(E^{\#}\right) \cap D\left(\mathscr{E}^{\#}\right)\right\}$. By 3.6 (iii) each $u \in D$ is $\mathscr{E}$-quasi-continuous and by 3.3 (iii) $D$ is $\widetilde{\mathscr{E}}^{1 / 2}$-dense in $D(\mathscr{E})$, and 2.8 (ii) is proved. Since $C_{0}\left(E^{\#}\right)$ is separable and $C_{0}\left(E^{\#}\right) \cap D\left(\mathscr{E}^{\#}\right)$ is dense in $C_{0}\left(E^{\#}\right)$ with respect to the uniform norm, we can find a countable family $\left\{u_{n}^{\#} \mid n \in \mathbf{N}\right\} \subset C_{0}\left(E^{\#}\right) \cap D\left(\mathscr{E}^{\#}\right)$ such that $\left\{u_{n}^{\#} \mid n \in \mathbf{N}\right\}$ separates the points of

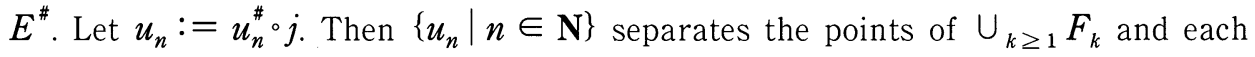
$u_{n}$ is an $\mathscr{E}$-quasi-continuous element of $D(\mathscr{E})$. Thus 2.8 (iii) is verified, completing the proof of the "if"-part.

(ii) "only if"-part (cf. [MR92, VI. 1. Local Compactification] or [AMR92b]): Suppose that $(\mathscr{E}, D(\mathscr{E}))$ is a quasi-regular Dirichlet form on $L^{2}(E ; m)$. Let $D$ be a countable dense subset of $D(\mathscr{E})$ specified by 2.10 , say $D:=\left\{u_{n} \mid n \in \mathbf{N}\right\}$ with $u_{1}:=h^{2}$. Let $\left(F_{k}\right)_{k \in \mathbf{N}}$ be specified by 2.10 (iii) and $Y:=\cup_{k \geq 1} F_{k}$. Set $g_{n}:=\frac{2}{\pi}$. $\arctan u_{n}$ and define a map $j: Y \rightarrow[0,1]^{\mathbf{N}}$ by $j(x):=\left(g_{n}(x)\right)_{n \in \mathbf{N}}$. Let $X^{\#}=j(Y)$. 
Since $\left\{g_{n} \mid n \in \mathbf{N}\right\}$ separates the points of $Y, j$ is a one-to-one map onto $Y^{\#}$. Let $\bar{Y}^{\#}$ be the completion of $Y^{\#}$ in $[0,1]^{\mathbf{N}}$ with respect to the metric $\rho$ defined by

$$
\rho\left(w, w^{\prime}\right):=\sum_{n \geq 1} 2^{-n}\left|w_{n}-w_{n}^{\prime}\right|, \text { if } w:=\left(w_{n}\right)_{n \in \mathbf{N}}, w^{\prime}:=\left(w_{n}^{\prime}\right)_{n \in \mathbf{N}} .
$$

Then $\bar{Y}^{\#}$ is a compact metric space. If $w:=\left(w_{n}\right)_{n \in \mathbf{N}} \in Y^{\#}$, then $g_{n}{ }^{\circ} j^{-1}(w)=w_{n}$. Therefore, all $g_{n} \circ j^{-1}$ and $u_{n}{ }^{\circ} j^{-1}$ are uniformly continuous on $Y^{\#}$. Let $u_{1}^{\#}$ be the unique extension of $u_{1}{ }^{\circ} j^{-1}$ to $\bar{Y}^{\#}$ and

$$
E^{\#}:=\left\{w \in \bar{Y}^{\#} \mid u_{1}^{\#}(w)>0\right\} .
$$

Then $E^{\#}$ is a locally compact separable metric space. Since $u_{1} \circ j^{-1}=h^{2} \circ j^{-1}$ is strictly positive on $Y^{\#}$, we have that $Y^{\#} \subset E^{\#}$. For all $n \in \mathbf{N}$, let $u_{n}^{\#}$, $g_{n}^{\#}$ be the unique continuous extensions to $E^{\#}$ of $u_{n}{ }^{\circ} j^{-1}, g_{n} \circ j^{-1}$, respectively. Let $F_{k}^{\#}:=$ $j\left(F_{k}\right)$. Since each $g_{n}$ is continuous on $F_{k}, j:=\left(g_{n}\right)_{n \in \mathbf{N}}$ is a continuous one-to-one map from $F_{k}$ onto $F_{k}^{\#}$. But by 2.10 (iii) $F_{k}$ is compact. Therefore, $F_{k}^{\#}$ is compact and $j$ is a homeomorphism from $F_{k}$ onto $F_{k}^{\#}$ which implies in turn that $Y^{\#}:=$ $\cup_{k \geq 1} F_{k}^{\#}$ is a Borel subset of $E^{\#}$. Let $m^{\#}:=m^{\circ} j^{-1}$ and extend $m^{\#}$ to $\mathscr{B}\left(E^{\#}\right)$ by setting $m^{\#}\left(E^{\#} \backslash Y^{\#}\right):=0$. Let $\left(\mathscr{E}^{\#}, D\left(\mathscr{E}^{\#}\right)\right)$ be the image $\left(\mathscr{E}^{j}, D\left(\mathscr{E}^{j}\right)\right.$ ) (cf. (3.2)) of $(\mathscr{E}, D(\mathscr{E}))$ under $j$. One can check that $D\left(\mathscr{E}^{\#}\right)$ is dense in $L^{2}\left(E^{\#} ; m^{\#}\right)$ and hence $\left(\mathscr{E}^{\#}, D\left(\mathscr{E}^{\#}\right)\right)$ is a Dirichlet form on $L^{2}\left(E^{\#} ; m^{\#}\right)$. If $u \in D(\mathscr{E})_{F_{k}}$, then $u \circ j^{-1} \in$ $D\left(\mathscr{E}^{\#}\right)_{F_{k}^{*}}$. Therefore, $U_{k \geq 1} D\left(\mathscr{E}^{\#}\right)_{F_{k}^{*}}$ is $\mathscr{\mathscr { E }}_{1}^{\# 1 / 2}$-dense in $D\left(\mathscr{E}^{\#}\right)$, which implies that $\left(F_{k}^{\#}\right)_{k \in \mathbf{N}}$ is an $\mathscr{E}^{\#}$-nest. What we have proved shows that $j$ is a quasihomeomorphism from $(\mathscr{E}, D(\mathscr{E}))$ to $\left(\mathscr{E}^{\#}, D\left(\mathscr{E}^{\#}\right)\right)$. It remains to show that $\left(\mathscr{E}^{\#}\right.$, $\left.D\left(\mathscr{E}^{\#}\right)\right)$ is regular. To this end we set

$$
\begin{array}{r}
C_{\infty}\left(E^{\#}\right):=\left\{u^{\#} \mid u^{\#} \text { is continuous on } E^{\#} \text { and }\left\{u^{\#} \geq \delta\right\} \text { is compact in } E^{\#}\right. \\
\text { for all } \delta>0\} .
\end{array}
$$

Let $D^{\#}:=\left\{u_{n}^{\#} \mid n \in \mathbf{N}\right\}$. Then $D^{\#}$ is $\widetilde{\mathscr{E}}_{1}^{\# 1 / 2}$-dense in $D\left(\mathscr{E}^{\#}\right)$. By 2.10 (ii) and (3.11) $D^{\#} \subset C_{\infty}\left(E^{\#}\right)$. Since $u_{1}^{\#} \in D^{\#}$ is strictly positive on $E^{\#}$ and $D^{\#}$ is an algebra over the rationals which separates the points of $E^{\#}$, by the Stone-Weierstraß Theorem $D^{\#}$ is dense in $C_{\infty}\left(E^{\#}\right)$ with respect to the uniform norm. Let $C_{0}\left(E^{\#}\right)$ be the family of all continuous functions with compact support on $E^{\#}$. Noting that for each $u^{\#} \in D^{\#}, u_{\delta}^{\#}:=u^{\#}-\left(\left(u^{\#} \vee(-\delta)\right) \wedge \delta\right) \in C_{0}\left(E^{\#}\right) \cap D\left(\mathscr{E}^{\#}\right)$ for all $\delta>0$, and $u_{\delta}^{\#} \rightarrow u^{\#}$ uniformly and with respect to $\widetilde{\mathscr{E}}_{1}^{\# 1 / 2}$ when $\delta \downarrow 0$, we conclude that $\left(\mathscr{E}^{\#}, D\left(\mathscr{E}^{\#}\right)\right)$ satisfies (3.8) and (3.9). Since $u_{1} \in L^{1}(E ; m)$, hence $u_{1}^{\#} \in L^{1}\left(E^{\#}\right.$; $\left.m^{\#}\right), m^{\#}$ is a Radon measure. The fact that $F_{k}=\operatorname{supp}\left[I_{F_{k}} \cdot m\right]$ implies that $\operatorname{supp}\left[m^{\#}\right]=E^{\#}$. Therefore $\left(\mathscr{E}^{\#}, D\left(\mathscr{E}^{\#}\right)\right)$ is regular. 
Remark 3.8. (i) In the second part of the above proof let us set

$$
\rho\left(x, x^{\prime}\right):=\sum_{n \geq 1} 2^{-n}\left|g_{n}(x)-g_{n}\left(x^{\prime}\right)\right| ; x, x^{\prime} \in Y .
$$

Let $\bar{Y}$ be the completion of $Y$ with respect to the $\rho$-topology. Let $\bar{u}_{1}$ be the continuous extension of $u_{1}$ to $\bar{Y}$ and define

$$
E^{\#}:=\left\{x \in \bar{Y} \mid \bar{u}_{1}(x)>0\right\} .
$$

Then we may construct a regular Dirichlet form on $E^{\#}$ quasi-homeomorphic to $(\mathscr{E}, D(\mathscr{E}))$. In this case we have even $Y \subset E^{\#}$. This was done in [MR92] (cf. also [AMR92b]) and was called local compactification of $E$. The local compactification method provides a procedure to transfer all results known for regular Dirichlet forms to the case of quasi-regular Dirichlet forms. For details see [MR92. Chap. VI]. In particular, as already mentioned in [MR92, Chap VI, Remark 2.6], it can be shown on the basis of the classical results [F71a], [Ca-Me75] for regular Dirichlet forms and the quasi-homeomorphism established in Theorem 3.7 that every quasi-regular Dirichlet form admits an associated strong Markov process-more precisely, an $m$-tight special standard process. This was first proved by a direct construction in [AMR92] and [AMR93], (see also [AMR92a]).

(ii) Starting from Proposition 2.10, one can also construct a regular Dirichlet form by using the Gelfand transform. This approach can be traced back to Fukushima's regular representation theory [F71b] and will be discussed in the next section.

\section{§4. Gelfand transform approach to local compactification}

This section contains an alternative proof of the "only if" part of Theorem 3.7. Suppose that $(\mathscr{E}, D(\mathscr{E}))$ is a quasi-regular Dirichlet form on $L^{2}(E ; m)$. Let $D=\left\{f_{n} \mid n \in \mathbf{N}\right\}$ be a dense subset of $D(\mathscr{E})$ specified by 2.10 and $\left(F_{k}\right)_{k \in \mathbf{N}}$ be an $\mathscr{E}$-nest satisfying 2.10 (iii). Henceforth any function $f \in D$ is assumed to be zero on $E \backslash Y$, where $Y:=\cup_{k \geq 1} F_{k}$. With this convention, functions in $D$ are uniquely defined everywhere on $E$ and $\|f\|_{\infty}$, the $L^{\infty}$ norm of $f$ on $E$, is just $\sup \{|f(x)| \mid$ $x \in Y\}$. The uniform closure $\bar{D}$ of $D$ is a commutative Banach algebra. Therefore, a regular Dirichlet form $\left(\mathscr{E}^{\#}, D\left(\mathscr{E}^{\sharp}\right)\right)$ on a locally compact separable metric space $E^{\#}$ can be constructed from $\bar{D}$ via Gelfand transform, which will turn out to be quasi-homeomorphic to the original Dirichlet form $(\mathscr{E}, D(\mathscr{E}))$.

Step 1. Construction of a locally compact separable metric space $E^{\#}$ 
Let $E^{\#}$ be a collection of non-trivial real valued functions $\gamma$ on $\bar{D}$ which satisfy for $f, g \in \bar{D}$ and for $a, b$ rational

$$
\begin{gathered}
|\gamma(f)| \leq\|f\|_{\infty}, \\
r(f g)=\gamma(f) \cdot \gamma(g), \\
\gamma(a f+b g)=a r(f)+b \gamma(g) .
\end{gathered}
$$

We equip $E^{\#}$ with the weakest topology such that the function $\Phi_{f}: \gamma \rightarrow f(\gamma)$ is cotinuous for each $f \in \bar{D}$. It is well known that $E^{\#}$ is a separable locally compact Hausdorff space which is compact if and only if $1 \in \bar{D}$ and $\left\{\Phi_{f}, f \in \bar{D}\right\} \subset$ $C_{\infty}\left(E^{\#}\right) . E^{\#}$ is metrizable with metric $d$ defined by

$$
d\left(\gamma, \gamma^{\prime}\right)=\sum_{n=1}^{\infty} \frac{\left|f_{n}(\gamma)-f_{n}\left(\gamma^{\prime}\right)\right| \wedge 1}{2^{n}}, \gamma, \gamma^{\prime} \in E^{\#} .
$$

Clearly, by (2.10) each point $x$ in $Y$ satisfies for $f, g \in D$ and for $a, b$ rational

$$
\begin{gathered}
|f(x)| \leq\|f\|_{\infty}, \\
(f g)(x)=f(x) \cdot g(x), \\
(a f+b g)(x)=a f(x)+b g(x) .
\end{gathered}
$$

Since $D$ is uniformly dense in $\bar{D},(4.4)-(4.6)$ are valid for all $f, g \in \bar{D}$. Let $j$ be the unique map from $Y$ to $E^{\#}$ such that

$$
(j x)(f)=f(x), \text { for } f \in \bar{D} \text { and } x \in Y .
$$

By (2.10), $j$ is a continuous one-to-one map on each $F_{n}$. Hence $F_{n}^{\#}:=j\left(F_{n}\right)$ is compact in $E^{\#}$ and $j$ is a homeomorphism from $F_{n}$ onto $F_{n}^{\#}$ for each $n \in \mathbf{N}$.

Since $j$ is Borel measurable, there is a unique Borel measure $m^{\#}$ on $E^{\#}$ such that

$$
\int_{E^{\#}} \phi(\gamma) m^{\#}(d \gamma)=\int_{Y} \phi(j x) m(d x)
$$

for nonnegative $\phi$ on $E^{\#}$. Clearly,

$$
m^{\#}\left(E^{\#} \backslash Y^{\#}\right)=0 \text {, where } Y^{\#}:=\bigcup_{k \geq 1} F_{k}^{\#} .
$$

It follows from the $m$-integrability of functions in $D$ that $m^{\#}$ is Radon and it is easy to check that $\operatorname{supp}\left[m^{\#}\right]=E^{\#}$ (see [Si74, p.23]). 
Step 2. $j$ maps $\bar{D}$ onto $C_{\infty}\left(E^{\#}\right)$

We use the same symbol $j$ to denote the natural mapping of $B$ into $C_{\infty}\left(E^{\#}\right)$; that is,

$$
(j f)(\gamma)=\gamma(f), \quad f \in \bar{D}, \gamma \in E^{\#} .
$$

Since $j(Y)=Y^{\#} \subset E^{\#}$,

$$
\|j f\|_{\infty}=\sup _{\gamma \in E^{\#}}|\gamma(f)| \geq \sup _{x \in Y}|j x(f)|=\sup _{x \in Y}|f(x)|=\|f\|_{\infty}, f \in \bar{D} .
$$

On the other hand by (4.1),

$$
\|j f\|_{\infty}=\sup _{\gamma \in E^{\#}}|j f(\gamma)|=\sup _{x \in E^{\#}}|\gamma(f)| \leq\|f\|_{\infty}, f \in \bar{D} .
$$

Therefore, $\|j f\|_{\infty}=\|f\|_{\infty}$ for $f \in \bar{D}$. So $j \bar{D}$ is closed with respect to the uniform norm. Since $j \bar{D}$ is an algebra of real-valued continuous which vanish at infinity and separate points on $E^{\#}$, by the Stone-Weierstraß Theorem, $j \bar{D}=C_{\infty}\left(E^{\#}\right)$. In particular, $j \bar{D}$ is uniformly dense in $C_{\infty}\left(E^{\#}\right)$.

Step 3. The induced regular Dirichlet form on $E^{\#}$ by $(\mathscr{E}, D(\mathscr{E}))$ via $j$ For a function $f$ which is m-a.e. defined on $E$, let $j f$ be the unique function on $E^{\#}$ modulo an $m^{\#}$-null set such that $j f(\gamma)=f\left(j^{-1} \gamma\right)$ for $\gamma \in Y^{\#}=j(Y)$. It follows from (4.8) that $j$ is an isometry from $L^{2}(E ; m) \cap \bar{D}$ onto $L^{2}\left(E^{\#} ; m^{\#}\right) \cap C_{\infty}\left(E^{\#}\right)$ and from $L^{2}(E ; m)$ onto $L^{2}\left(E^{\#} ; m^{\#}\right)$. Clearly the mapping $j$ has the following property:

$$
j(0 \vee f \wedge 1)=0 \vee j(f) \wedge 1 m^{\#} \text {-a.e. on } E^{\#}
$$

for any Borel function $f$ on $E$. Let

$$
D\left(\mathscr{E}^{\#}\right)=j(D(\mathscr{E}))=\{j f \mid f \in D(\mathscr{E})\},
$$

and

$$
\mathscr{E}^{\#}\left(f^{\#}, f^{\#}\right)=\mathscr{E}(f, f), \quad \text { if } \quad j f=f^{\#} .
$$

Then $\left(\mathscr{E}^{\#}, D\left(\mathscr{E}^{\#}\right)\right)$ is a Dirichlet form on $L^{2}\left(E^{\#} ; m^{\#}\right)$. Since $D\left(\mathscr{E}^{\#}\right) \cap C_{\infty}\left(E^{\#}\right)$ $(\supset j D)$ is uniformly dense in $C_{\infty}\left(E^{\#}\right)$ and $\widetilde{\mathscr{E}}_{1}^{\# / 2}$-dense in $D\left(\mathscr{E}^{\sharp}\right)$ (because $D$ is dense in $D(\mathscr{E})),\left(\mathscr{E}^{\#}, D\left(\mathscr{E}^{\#}\right)\right)$ is a regular Dirichlet form on $L^{2}\left(E^{\#} ; m^{\#}\right)$. Note that $j$ is an isometry from $(\mathscr{E}, D(\mathscr{E}))$ onto $\left(\mathscr{E}^{\#}, D\left(\mathscr{E}^{\#}\right)\right)$. Similarly to the discussion in the previous section, we can now show that $\left(F_{n}^{\#}\right)_{n \in \mathbf{N}}$ is an $\mathscr{E}^{\#}$-nest and hence $j$ is a quasi-homeomorphism from $(\mathscr{E}, D(\mathscr{E}))$ to $\left(\mathscr{E}^{\#}, D\left(\mathscr{E}^{\#}\right)\right)$. 
Acknowledgement. We would like to thank S. Albeverio and P.J. Fitzsimmons for very helpful discussions on this work. The second-named author would like to thank his hosts M. Silverstein and Z.Q. Chen for the hospitality during a very pleasant stay at Washington University in St. Louis. Financial support of the Chinese National Science Foundation, SFB 256 Bonn and the Max-PlanckGesellschaft is gratefully acknowledged.

\section{REFERENCES}

[AM91] Albeverio, S., Ma, Z. M., Necessary and sufficient condition for the existence of $\mathrm{m}$-perfect processes associated with Dirichlet forms, In: Seminaire de Probabilités XXV, Lecture Notes in Math., 1485, 374-406, Berlin: Springer 1991.

[AM92] - A general correspondence between Dirichlet forms and right processes, Bull. Amer. Math. Soc., 26 (1991), 245-252.

[AMR90] Albeverio, S., Ma, Z. M., Röckner, M., A Beurling-Deny type structure theorem for Dirichlet forms on general state space, Preprint (1990), In: Memorial Volume for R. Høegh-Krohn, Vol. I. Ideas and methods in mathematical analysis, stochastics and applications, Ed. S. Albeverio, J. E. Fenstad, H. Holden, T. Lindstrøm. Cambridge: Cambridge University Press (1992).

[AMR92a] - Non-symmetric Dirichlet forms and Markov processes on general state space, C. R. Acad. Sci. Paris, t. 314 Série I (1992), 77-82.

[AMR92b] - Regularization of Dirichlet spaces and applications, C. R. Acad. Sci. Paris, t. 314, Série I (1992), 859-864.

[AMR93] - Quasi-regular Dirichlet forms and Markov processes, J. Funct. Anal., 111 (1993), 118-154.

[Ca-Me75] Carrillo Menendez, S., Processus de Markov associé à une forme de Dirichlet non symétrique, Z. Wahrsch. verw. Geb., 33 (1975), 139-154.

[F71a] Fukushima, M., Dirichlet spaces and strong Markov processes, Trans. Amer. Math. Soc., 162 (1971), 185-224.

[F71b] - Regular representations of Dirichlet forms, Amer. Math. Soc., 155 (1971), 455-473.

[F80] Fukushima, M., Dirichlet forms and Markov processes, AmsterdamOxford-New York: North Holland (1980).

[Le77] LeJan,Y., Balayage et formes de Dirichlet, Z. Wahrsch. verw. Geb., 37 (1977), 297-319.

[MR92] Ma, Z. M., Röckner, M., An introduction to the theory of (non-symmetric) Dirichlet forms, Berlin: Springer 1992.

[Si74] Silverstein, M. L., Symmetric Markov Processes, Lecture Notes in Math., 426, Berlin-Heidelberg-New York: Springer 1974. 
Z.-Q. Chen.

Department of Mathematics

University of California at

San Diege

La Jolla, CA 92093

USA

Z.-M. Ma

Institute of Applied Mathematics

Academia Sinica

100090 Beijing

China

M. Röckner

Faculty of Mathematics

University of Bielefeld

D-33501 Bielefeld

Germany 